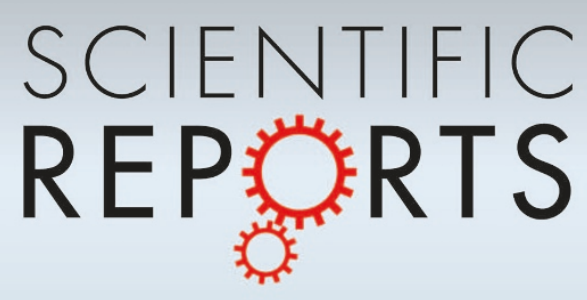

OPEN

SUBJECT AREAS:

STATISTICAL PHYSICS, THERMODYNAMICS AND NONLINEAR DYNAMICS

POLYMERS AND SOFT MATERIALS

GEOPHYSICS

APPLIED PHYSICS

Received

11 June 2012

Accepted

17 July 2012

Published

2 August 2012

Correspondence and requests for materials should be addressed to D.B. (d.bonn@uva.nl)

\section{How to construct the perfect sandcastle}

\author{
Maryam Pakpour ${ }^{1,2}$, Mehdi Habibi ${ }^{1}$, Peder Møller ${ }^{3}$ \& Daniel Bonn ${ }^{2,3}$
}

${ }^{1}$ Institute for Advanced Studies in Basic Sciences, P.O. Box 45 195-1 159, Zanjan, Iran, ${ }^{2}$ Van der Waals-Zeeman Institute, loP, University of Amsterdam, Science Park 904, 1098XH, Amsterdam, the Netherlands and, ${ }^{3}$ Laboratoire de Physique Statistique de I'ENS, 24 Rue Lhomond, 75231 Paris Cedex 05, France.

Just a bit of water enables one to turn a pile of dry sand into a spectacular sandcastle. Too much water however will destabilize the material, as is seen in landslides. Here we investigated the stability of wet sand columns to account for the maximum height of sandcastles. We find that the columns become unstable to elastic buckling under their own weight. This allows to account for the maximum height of the sand column; it is found to increase as the $2 / 3$ power of the base radius of the column. Measuring the elastic modulus of the wet sand, we find that the optimum strength is achieved at a very low liquid volume fraction of about $1 \%$. Knowing the modulus we can quantitatively account for the measured sandcastle heights.

T he formation of capillary bridges between sand grains are the cause of the stiffness of sculptured wet sand in a sandcastle, as opposed to dry sand which can hardly or not support its own weight ${ }^{1}$. Qualitatively, the liquid leads to the formation of capillary bridges between the sand grains, and the curvature of the liquid interface leads to a capillary pressure causing a force of attraction between the grains. This then creates a network of grains connected by pendular bridges, and allows, for example, creating complex structures such as sandcastles. Not many quantitative studies on the mechanical properties of wet sand exist, in spite of the fact that the handling and flow of granular materials is responsible for roughly $10 \%$ of the world energy consumption ${ }^{2}$.

Since in many cases the humidity in the air is sufficient for liquid bridges to form between sand grains, one would expect the mechanical behavior to be well known. This is not the case, in spite of the fact that the stability of wet granular packings is of paramount importance for civil engineering purposes and that the adhesive forces due to the presence of liquid bridges are also extremely important in geophysical applications (i.e., soil stability), of which sandcastles are merely an unusual example ${ }^{3-8}$. For sandcastles, the only estimate in the literature ${ }^{9}$, argues that the stability is related to the capillary rise in the granular medium, and arrives at a maximum sandcastle height of roughly $20 \mathrm{~cm}$. This is in stark disagreement with the observation of sandcastles of several meters high, and the common observation that the stability depends on the base radius of the sand structure.

To account for the (in)stability of sandcastles, we show here that it is sufficient to consider that the limit of instability is reached when a column of sand undergoes a buckling transition under its own weight. An elastic rod becomes elastically unstable and buckles under its own weight when exceeding a critical height $h_{\text {crit }}{ }^{10}$. We present here the analytical solution for $h_{\text {crit }}$ for a cylindrical column:

$$
h_{\text {crit }}=\left(\frac{9 J_{(-1 / 3)}^{2}}{16} \frac{G R^{2}}{\rho g}\right)^{1 / 3},
$$

where $G$ is the elastic modulus, $R$ the column radius, $\rho$ is density, $g$ the gravitational acceleration, and $J \approx 1.8663$ is the smallest positive root of the Bessel function of the first kind of order $-1 / 3^{11}$. A similar expression is used in civil engineering to calculate the stability of buildings ${ }^{12}$ and we therefore expect that this also gives the maximum height for which a sandcastle falls apart as the buckling instability will cause the sandcastle to fracture.

\section{Results}

From the buckling arguments, the maximum height varies with the base radius as $h_{\text {max }} \sim R^{2 / 3}$. The experimental data for the maximum height as a function of the column radius compare rather favorably with the theoretical expression for buckling (Fig. 2). The exponent of experimental data is in good agreement with theory: $h_{\exp } \sim$ $R^{0.7 \pm 0.05}$

To be able to quantitatively compare with the buckling prediction for the maximum height of a sandcastle, we need to quantify the shear modulus. A recently introduced model for the strength of wet granular matter ${ }^{13}$ assumes that when one adds a volume of liquid to grains, the capillary attractive force and elastic response from the Hertz contact between two spheres will be balanced. As two beads are always separated by at least the surface 


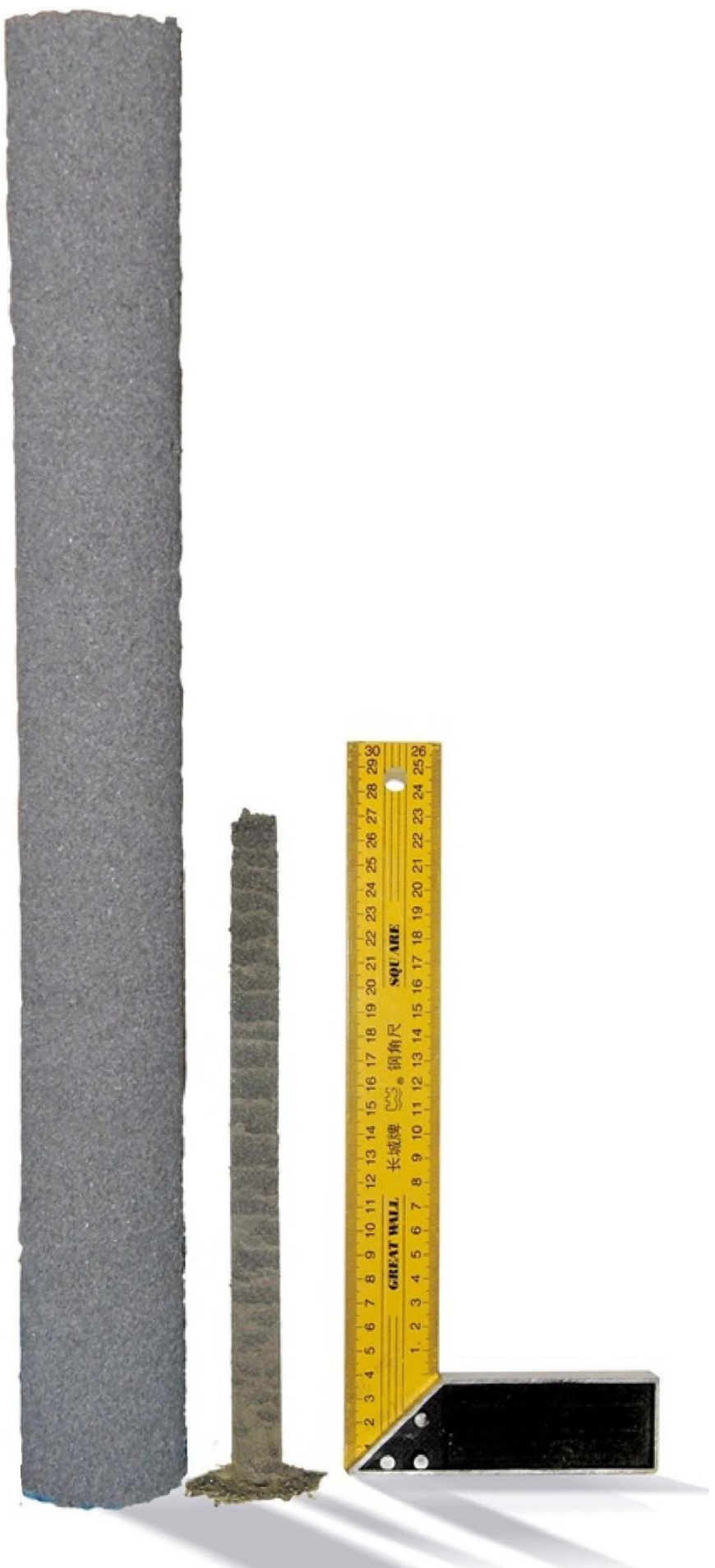

Figure 1 Sandcastles with diameters $2 \mathrm{~cm}$ and $7 \mathrm{~cm}$.

roughness, below a critical liquid volume fraction about $0.2 \%$, the bridges between the beads cannot form. At higher volume fractions, the bridge force is dominated by the curvature of the meniscus and at even higher volume fractions the bridges start merge into larger pockets of fluid ${ }^{13}$. The macroscopic shear modulus $G$ of a macroscopic cube of dimension $L$ containing a large amount of grains can be defined as the ratio of stress and strain:

$$
G=2(1+v) \frac{F_{\text {strain }} / L^{2}}{\Delta x / L},
$$

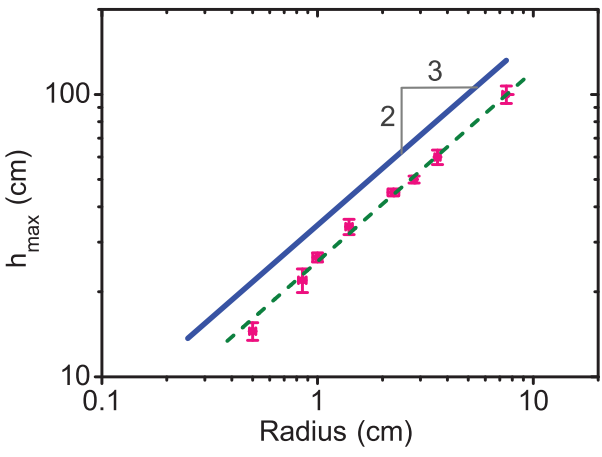

Figure $2 \mid$ Experimental data points and theoretical prediction of the maximum height of sandcastle as a function of its radius. The solid line is the theory without any adjustable parameters using $G=0.054 a^{-1 / 3} E^{2 / 3} \gamma^{1 / 3}$, where $a=100 \mu \mathrm{m}, E=30 \mathrm{GPa}, \gamma=70 \mathrm{mN} / \mathrm{m}$. The density of the sand is $\rho=2.6 \mathrm{~g} / \mathrm{cm}^{3}$. The small but systematic discrepancy between the theory and the experiments is likely to be due to perturbations that arise when we remove the PVC pipes, leading to a somewhat smaller maximum height than the theoretical one.

where $\Delta x / L$ is the strain, $F_{\text {strain }} / L^{2}$ the stress and $v \approx 0.5$ the Poisson ratio. Assuming that at the level of single particles the capillary and elastic forces are balanced for each pair of grains and using the simple Hertz contact for the grain contact elasticity, the optimum strength $G$ can be found by averaging over all pairs as ${ }^{13}$ :

$$
G=\alpha a^{-1 / 3} E^{2 / 3} \gamma^{1 / 3},
$$

where $a$ is the radius of the grains, $E$ is the Young's modulus of the grain material and $\gamma$ is the surface tension of the liquid-air interface, respectively. $\alpha$ is constant of proportionality that expresses how much the individual capillary sphere-sphere bonds are deformed relative to the globally imposed strain. To compute an estimate of $\alpha$, we take a simple cubic crystal of frictionless spheres and average $\alpha$ over different straining directions, which gives $\alpha \approx 0.054$. We take the function $f\left(V_{f}\right) \approx 1$ for determining the maximum strength; $f\left(V_{f}\right)$ shows the dependence of the elastic modulus to the liquid volume fraction and is unity for the optimum volume fraction ${ }^{13}$.

\section{Discussion}

This model gives a very accurate result for the maximum strength of the sand packing (Fig. 3), which makes it possible to compute how high sandcastles can be built from the predicted elastic modulus for any size grains of wet sand. Using typical values for beach sand, a

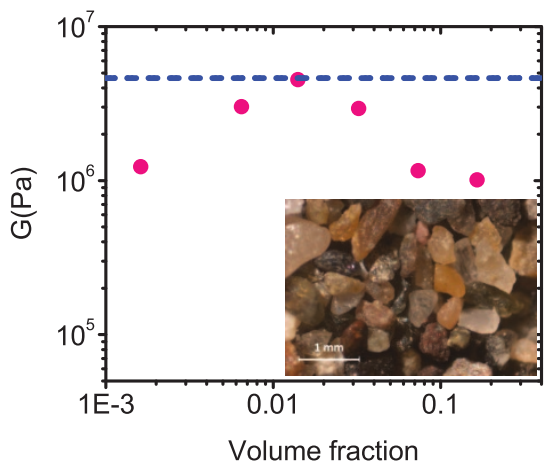

Figure 3 The elastic shear modulus vs. volume fraction of water (circles: experimental measurements, dashed line: theoretical prediction for the optimal strength from Eq. 3). The measurements were done on a commercial rheometer using a vane-in cup geometry where the cup was covered with sandpaper and the sand compacted as for the sandcastle experiments. Inset: picture of the grains. 


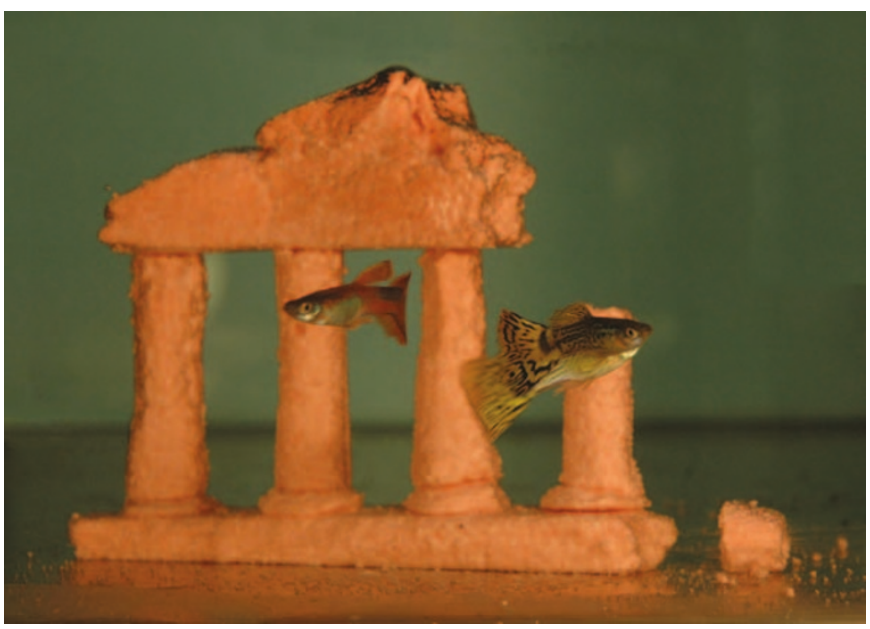

Figure $4 \mid$ An underwater sandcastle. Using commercially available hydrophobic sand it is possible to build an underwater sandcastle. Since the force between beads remain constant, but the effective weight of the sandcastle is reduced by a factor of 3 , it is possible to build more spectacular sandcastles underwater than above. The different elements of this sandcastle were molded under water, saturated with interstitial air. After molding, a syringe was used to suck out air from the elements, reducing the "fluid" volume fraction from about $40 \%$ to about $10 \%$ in order to increase the strength of the material before simply moving them into place by hand.

cylinder with a radius of $20 \mathrm{~cm}$ for instance could be as tall as about $2.5 \mathrm{~m}$, which is in quite good agreement with what can be observed for real sandcastles ${ }^{14}$. This estimate is an immense improvement compared to a previous dimensional analysis result which gives roughly $20 \mathrm{~cm}$ as the maximum height of a sandcastle, independently of the base diameter ${ }^{9}$. For our cylindrical sandcastles, using the optimum strength in the buckling arguments, we arrive at a quantitative theory for sandcastle stability that agrees with the measurements (Fig. 2).

Can we use these new insights to build taller sandcastles? From $h_{\max }$ we see that besides the sandcastle diameter, the most potent power is associated with $\alpha / \rho g$. We cannot change $g$, but $\alpha$ can be increased by compaction which is always done by sandcastle builders. Also we could decrease the effective density, $\rho$, of our sandcastle by plunging it under water. For normal sand however, this will destroy the liquid bridges between grains and thus the strength of the material. However, if hydrophobic sand is used the roles of water and air interchange completely ${ }^{15}$. In this case the air and not the water "wets" the grains and we can simply interchange water and air, which does not change the bridge force. Since the force between beads remain constant, but the effective density of compacted sand changes from $1.6 \mathrm{~g} / \mathrm{cm}^{3}$ to $0.6 \mathrm{~g} / \mathrm{cm}^{3}$ when immersed in water. This makes it possible to build underwater sandcastles, which are even more spectacular than normal ones (Fig. 4).
These results are of practical interest for civil engineering and soil mechanics, as well as fundamental interest to come to a better understanding of partially saturated granular materials. In addition it explains the maximum height of, and provides us with a recipe to construct the perfect sandcastle.

\section{Methods}

To verify this experimentally, beach sand with an average radius of $100 \mu \mathrm{m}$ was mixed with a small amount of deionized water. Cylindrical 'sandcastles' were constructed using non-wetting PVC pipes of different diameters cut in half over the length of the tube. The two halves were assembled, and the wet sand was put in the tube standing on vertically on a surface. The wet sand was poured into the pipe in small portions and compacted by dropping a thumper into the pipe at least 70 times. This process was repeated until the pipe was filled with sand up to a certain height. The two halves of the cylindrical tube were then carefully removed and if the sand column was stable, a new experiment was launched filling the tube to a larger height, until the column collapsed. Several experiments were done at each filling height to ensure the reproducibility of the results. Figure 1 shows two columns of sand with height $27 \mathrm{~cm}$ and $60 \mathrm{~cm}$ with diameters $2 \mathrm{~cm}$ and $7 \mathrm{~cm}$. This procedure was followed for 8 pipes of diameter ranging between 0.5 and 7.5 centimeters.

1. Kudrolli, A. Sticky sand. Nature Mater. 7, 174-175 (2008).

2. Durand, J. Sands, Powders and Grains (Springer Verlag, 2000).

3. Nowak, S. et al. Maximum angle of stability of a wet granular pile. Nature Phys. 1, 50-52 (2005).

4. Scheel, M. et al. Morphological clues to wet granular pile stability. Nature Mater. 7, 189-193 (2008).

5. Hornbaker, D. J. et al. What keeps sandcastles standing. Nature 387, 765 (1997).

6. Schiffer, P. A bridge to sandpile stability. Nature Phys. 1, 21-22 (2005).

7. Tegzes, P. et al. Avalanche dynamics in wet granular materials. Phys. Rev. Lett. 89, 094301 (2002).

8. Halsay, T. C. \& Levine, A. J. How Sandcastles fall. Phys. Rev. Lett. 80, 3141 (1998).

9. Quere, D. Non-sticking drops. Rep. Prog. Phys. 68, 24952532 (2005).

10. Landau, L. D. \& Lifshitz, E. M. Theory of Elasticity (Pergamon Press, 1970).

11. Cox, S. J. \& McCarthy, C. M. The shape of the tallest column. SIAM J. Math. Anal. 29, 547-554 (1998).

12. Pippard, A. J. S. The Analysis of Engineering Structures (E. Arnold and Co, 1950).

13. Moller, P. \& Bonn, D. The shear modulus of wet granular matter. Europhys.Lett. 80, 38002 (2007).

14. www.sandworld.de

15. Vitz, E. Magic sand: modeling the hydrophobic effect and reversed-phase liquid chromatography. Jour. Chem. Edu. 67, 512 (1990).

\section{Author contributions}

MP performed the experiments. MP prepared figure 1, MP and MH prepared figure 2, DB prepared figure 3, PM prepared figure 4 . All authors did the theoretical work and reviewed the manuscript.

\section{Additional information}

Competing financial interests: The authors declare no competing financial interests.

License: This work is licensed under a Creative Commons

Attribution-NonCommercial-ShareAlike 3.0 Unported License. To view a copy of this license, visit http://creativecommons.org/licenses/by-nc-sa/3.0/

How to cite this article: Pakpour, M., Habibi, M., Møller, P. \& Bonn, D. How to construct the perfect sandcastle. Sci. Rep. 2, 549; DOI:10.1038/srep00549 (2012). 